\title{
La evaluación de la persistencia basada en una tarea de aprendizaje adquisición-extinción
}

\section{The assessment of persistence based on an acquisition-extinction learning task}

\author{
José Manuel Hernández López ${ }^{1}$, José Héctor Lozano Bleda ${ }^{2}$, José Santacreu Mas ${ }^{1}$ \\ ${ }^{1}$ Universidad Autónoma de Madrid, España. ${ }^{2}$ Universidad Camilo José Cela, España.
}

Disponible online 30 de abril de 2011

\begin{abstract}
El presente trabajo tiene por objeto diseñar una prueba objetiva para la evaluación de la persistencia como variable de personalidad. Se analizan las condiciones para la evaluación de la persistencia en el comportamiento de acuerdo con los conceptos de persistencia motivacional, persistencia obcecación y persistencia exploración. Se propone como posible contexto para la evaluación de la persistencia un procedimiento de condicionamiento operante libre con fases de adquisición y extinción. Los resultados muestran que la prueba diseñada permite medir en la muestra estudiada las tres variables de persistencia. Los índices de consistencia interna son .928, para persistencia motivacional, .683 y .580 para los dos indicadores de persistencia obcecación y .905 para persistencia exploración. Las relaciones de cada una de las variables de persistencia con la motivación inicial de los participantes en la prueba son coherentes con la literatura.
\end{abstract}

Palabras clave: persistencia, evaluación objetiva de la personalidad, condicionamiento operante, extinción.

The aim of the present study was to design an objective task to assess persistence as a personality variable. The conditions for assessing behavioral persistence were analyzed according to the concepts of motivational persistence, obstinate persistence and exploratory persistence. A free operant procedure with an acquisition and an extinction phase was used as a possible context for assessing persistence. The results show that the designed task enabled the measurement of the three variables of persistence in the sample studied. Internal consistency indexes were .928 for motivational persistence, .683 and .580 for the two indicators of obstinate persistence, and .905 for exploratory persistence. The relationships between each persistence variable and the initial motivation of the participants are consistent with those described in the literature.

Keywords: persistence, objective personality assessment, operant conditioning, extinction.

Correspondencia: José Manuel Hernández López. Dpto. Psicología Biológica y de la Salud. Facultad de Psicología. Universidad Autónoma de Madrid. C7 Ivan Pavlov, 6. 28049 Madrid. E-mail: josemanuel.hernandez@uam.es. Teléfono: 9149787 52. Fax: 9149752 51. E-Mail de los otros autores: José Héctor Lozano Bleda: jhlozano@ucjc.edu; José Santacreu Mas: jose.santacreu@uam.es 


\section{La medición objetiva de la persistencia}

La principal función de las teorías de la personalidad es la de proponer constructos científicos que describan y expliquen la individualidad psicológica, es decir, la variabilidad en el comportamiento de los individuos ante una determinada tarea o situación (McAdams y Pals, 2007). La persistencia motivacional podría constituir uno de tales constructos en la medida en que representa un estilo de comportamiento consistente y estable.

Partiendo de la consideración de la persistencia en tanto que variable de personalidad, nuestro propósito es medirla de manera objetiva, esto es, de manera independiente del informe subjetivo del evaluado y del evaluador (Cattell, 1979). La evaluación objetiva de la persistencia exigiría el cumplimiento de los requerimientos propios de un proceso de evaluación, a saber: a) las características de la prueba no deben alterar el estilo de comportamiento que se pretende medir, omitiendo toda información relativa a la bondad de las respuestas y/o los criterios de actuación; y, b) tratándose de una variable de personalidad, se ha de controlar el efecto de las competencias y la motivación para llevar a cabo la tarea (Santacreu, Hernández, Adarraga y Márquez, 2002).

El tipo de situación general con que tradicionalmente se ha evaluado la persistencia en humanos consiste en enfrentar a una persona con una tarea irresoluble, sin restricción relativa al tiempo o al número de intentos para resolverla (Feather, 1962). En tal situación, el individuo podrá persistir en sucesivos intentos de solucionar la tarea, considerándose dicho número de intentos indicador de la persistencia del individuo. Resulta imprescindible, para poder evaluar la persistencia, que resulte creíble la posibilidad de una solución, aunque de hecho ésta nunca se logre. La situación planteada se corresponde con una situación experimental en la que no existe ninguna respuesta asociada a la consecuencia deseada. Tal situación es parecida a la de extinción de una determinada conducta previamente aprendida. En este sentido, el paradigma de aprendizaje operante, con fases de adquisición y extinción, podría representar una tarea útil para la evaluación de la persistencia (véase Santacreu y García-Leal, 2000). Así, durante la fase de adquisición, el individuo aprendería una serie de comportamientos que permitirían solucionar la tarea. En cambio, durante la fase de extinción, esos mismos comportamientos no lograrían el objetivo deseado.

La evaluación objetiva de la persistencia se basa en la observación del comportamiento de los individuos al tratar de resolver una tarea que no tiene solución. Tal observación aporta conocimiento sobre el número de intentos que se realizan. Sin embargo, una tarea operante de adquisición-extinción permitiría aportar datos adicionales respecto a las características de los intentos de solución, esto es, si se corresponden con antiguos comportamientos que previamente tuvieron éxito o si se trata de comportamientos novedosos.
El presente trabajo trata de explorar la posibilidad de evaluar la persistencia en un tiempo relativamente corto, con una nueva tarea de aprendizaje que implica un contexto de operante libre con fases de adquisición y extinción, similar a la tarea planteada por el Sistema Pavlov (Hernández, García-Leal, Rubio y Santacreu, 2004; Santacreu y García-Leal, 2000).

\section{Los tres componentes de la persistencia en una tarea de extin- ción}

El término persistencia hace referencia a repetir e intentar de nuevo. Se asume que, en todos los casos, dicha repetición es debida al fracaso en los intentos previos de solución del problema. La cuestión es diferenciar qué clase de comportamientos repetimos al realizar una tarea. Podríamos considerar que todo tipo de actividad constituye un intento de solución. En este sentido, como indicadores de la actividad se han propuesto medidas de frecuencia o tasa de todo comportamiento que pueda considerarse un nuevo intento de solución. Sin embargo, hemos de admitir que dichos indicadores también se han considerado medidas de la motivación de los individuos. Otra opción sería distinguir aquellos comportamientos que fueron la solución en otros contextos y/o momentos de los comportamientos novedosos. Así pues, la actividad mantenida en el tiempo con el objetivo de resolver tareas constituye un indicador de la persistencia motivacional.

A pesar de que la persistencia en el comportamiento se ha considerado adaptativa, no en toda circunstancia habría necesariamente de serlo. Así por ejemplo, en contextos, en los cuales la meta deseada fuera inalcanzable o las soluciones intentadas fueran ineficaces, un comportamiento persistente conduciría a un desajuste adaptativo. Consideraremos dos supuestos: a) la persistencia en el comportamiento que, pudiendo haber resultado útil en un contexto o momento distintos, ya se ha mostrado ineficaz en el contexto y tiempo presentes; este componente se correspondería con la denominada resistencia a la extinción y las muestras de mayor persistencia no deberían de considerarse adaptativas (Jenkins y Stanley, 1950; Lewis, 1960); y, b) la persistencia en el comportamiento basado en la emisión de nuevas respuestas que suponen un intento de encontrar una solución eficaz y novedosa a la situación, componente éste más próximo a la conducta de exploración (Berlyne, 1960; Hutt, 1970; Neuringer, 2002; Sutton y Barto, 1998).

Tal distinción entre repetir viejas soluciones versus intentar soluciones alternativas se relaciona con los términos explotación y exploración empleados en investigación sobre condicionamiento operante (Dam y Körding, 2009; Kaelbling, Littman y Moore, 1996; Sutton y Barto, 1998). La explotación, por una parte, se refiere a la emisión de comportamientos previamente aprendidos, conducentes a la obtención de reforzadores. En cambio, la exploración alude al comportamiento dirigido a aprender del entorno con el cual se está interactuando, con objeto de encontrar nuevas formas (o formas eficaces) de conse- 
guir refuerzo. El concepto de explotación está relacionado con la repetición y la estereotipia y el concepto de exploración con la variabilidad comportamental del individuo. De este modo, en entornos nuevos, con un nivel elevado de incertidumbre o en los que se obtienen bajas tasas de refuerzo, el comportamiento más adaptado sería el basado en la exploración. Ahora bien, en la medida en que el individuo permanece en el mismo contexto, aprende y obtiene refuerzo, la conducta más adaptada será repetir las respuestas previamente reforzadas, es decir, la solución que anteriormente resultó más eficaz. El comportamiento que el individuo repite es, por tanto, fruto del aprendizaje (la asociación R-C) y, naturalmente, explota los conocimientos del individuo sobre el contexto en que se aprendió (Daw, Niv y Dayan, 2005).

Los términos explotación y exploración han tenido también una gran repercusión dentro del ámbito organizacional, donde el primero alude al uso de conocimientos que ya se han mostrado útiles en el pasado y el segundo a la investigación, innovación y aprendizaje de nuevos conocimientos, que posteriormente se podrán explotar (Katila y Ahuja, 2002; March, 1991).

En resumen, podríamos distinguir tres componentes distintos de la persistencia: persistencia motivacional, persistencia obstinación y persistencia exploración.

El primer componente, la persistencia motivacional, está ligado a la actividad general y, por tanto, a la motivación. Hace referencia al interés o voluntad por llevar a cabo la tarea con éxito. La persistencia motivacional estaría relacionada con el número de intentos que, sin demora, se llevan a cabo por solucionar la tarea, estudiando la evolución de la tasa de respuesta durante el tiempo que dura la misma. En este sentido, el indicador más adecuado lo constituiría la tasa global de respuesta (Balleine, Garner, González y Dickinson, 1995; Honig y Staddon, 1975), considerando cada respuesta como un nuevo intento de solución.

El segundo componente, la persistencia obstinación, iría referido a los intentos de solución de una tarea mediante formas previas de solución de la misma. Este componente alude a una conceptualización de la persistencia como el mantenimiento de una secuencia de comportamientos que previamente conducía al éxito (Campbell y Pritchard, 1983). Las referencias inmediatas a este tipo de persistencia son los términos tozudez, obstinación, rigidez y empecinamiento, términos todos ellos que entroncan con el fenómeno de la estereotipia y la repetición (Moreno y Hunziker, 2008; Neuringer 2002). La persistencia obstinación estaría a su vez relacionada con el fenómeno de la insensibilidad a las contingencias, en el sentido en que el individuo se empecina y no atiende a las nuevas contingencias del contexto, probablemente porque sigue reglas o instrucciones que especifican una solución incoherente con las contingencias actuales (Catania, 1992; Joyce, y Chase, 1990; Luciano, 1993; Martínez y Tamayo, 2005). El adjetivo tozudo, se aplica a una persona que, a pesar de comprobar que una determinada solución no resulta eficaz en la situación y momento presentes, sigue probando de la misma manera, tardando en convencerse de que ya no resulta eficaz. En este sentido, el componente de obstinación se correspondería con la conducta de explotación en un contexto en el cual, el comportamiento previamente eficaz, o bien ha dejado de serlo en este momento, o bien no lo es porque se trata de un contexto diferente. El indicador podría ser el número, la tasa o la proporción de respuestas que previamente eran correctas respecto del total de las ejecutadas para resolver el problema. Dicho indicador exige una fase de adquisición y una fase de extinción, en la cual se registre la medida. Este componente implica un sentido negativo, por cuanto se intentan soluciones únicamente por el hecho de que previamente resultaban eficaces. Sin embargo, podemos también encontrarle un sentido positivo, en tanto una alta frecuencia de la respuesta previamente correcta indica un alto grado de aprendizaje previo, pero dicho sentido positivo únicamente quedaría patente si esta persistencia decae rápidamente con el tiempo. Por ello, este indicador de obstinación cobra fuerza en la medida en que el individuo persiste, es decir, en la medida en que la respuesta no decrece con el paso del tiempo.

Finalmente, el tercer componente constituye la característica positiva de la persistencia. Este componente alude al hecho de descartar rápidamente antiguas soluciones, ahora ineficaces, para concentrarse en la búsqueda de nuevas soluciones. Dicho comportamiento implica persistir en la exploración de nuevas alternativas en un contexto en el cual las conductas previamente aprendidas ya no resultan útiles. El problema es que la persistencia en los comportamientos previos es función de cuan exhaustivamente se hayan aprendido las contingencias. Cuando un individuo conoce con detalle en qué momento y bajo qué condiciones un comportamiento resulta eficaz, (cuanto más exhaustivamente conoce) le resulta más fácil, durante los primeros fracasos, comprender la inutilidad de dicho comportamiento y, en consecuencia, pasar a explorar otras alternativas. El número, proporción o tasa de respuestas nuevas y distintas sería un índice de la búsqueda y exploración perseverante.

\section{Objetivos del estudio}

La condición previa e imprescindible para obtener los dos últimos indicadores es que durante la fase de adquisición se produzca un aprendizaje, lo más exhaustivo posible, de las respuestas apropiadas. El trabajo previo, anteriormente citado (Hernández et al., 2004), planteaba una tarea en la que sólo el $18.95 \%$ de los participantes alcanzaban el criterio de aprendizaje estipulado para la fase de adquisición. Ello justifica el diseño de una nueva tarea que facilite el aprendizaje. Si tal diseño es acertado, permitirá, no sólo evaluar la persistencia motivacional, como en las previas de esta tarea, sino la persistencia relativa a la obstinación versus la persistencia de búsqueda y exploración.

En este sentido, el primer objetivo de este trabajo consiste en diseñar una tarea de aprendizaje que permita que un alto 
porcentaje de los participantes aprenda la conducta adecuada (reforzada) durante los ensayos programados para la fase de adquisición. Para ello, la tarea ha de ser relativamente sencilla, pero no obvia. Unido a esto, y dado que el objetivo es que se cumpla el criterio de aprendizaje para que todos los participantes puedan ser evaluados en la variable persistencia, el momento preciso en que el aprendizaje tenga lugar (que unos individuos aprendan antes que otros) no habría de afectar a los indicadores correspondientes a los tres componentes de la persistencia.

El segundo objetivo sería analizar las relaciones entre los tres componentes de la persistencia bajo la hipótesis de que la persistencia motivacional sería independiente de los otros dos componentes y que éstos se relacionarán entre sí de forma negativa debido a la incompatibilidad de los comportamientos indicadores de ambos.

Por otro lado, si bien la tasa de respuesta durante la fase de adquisición es, en parte, función de la tasa de refuerzo, también es función del grado de motivación del individuo, por lo que cabe hipotetizar que la motivación inicial mostrada por los individuos al aprender la tarea, correlacione positivamente con la persistencia motivacional mostrada durante la fase de extinción (aún cuando la tasa de respuesta en la fase de extinción sea mayor que en la fase de adquisición como efecto del estallido propio a la extinción). Esta hipótesis es acorde con estudios relativos a diferentes ámbitos (p. ej., académico, deportivo, etc.) que ponen de manifiesto que la motivación inicial es predictora de la persistencia del comportamiento (Allen, 1999; Cardoza, 1991; Matsumoto y Obana, 2001; Ramage, 1990), especialmente en el caso de la motivación intrínseca (Deci, Koestner y Ryan, 1999; Pelletier, Fortier, Vallerand y Briere, 2001; Vallerand y Bissonnette, 1992). Vollmeyer y Rheinberg (1998) sugieren en su modelo que la persistencia, como variable de personalidad, media la relación entre motivación inicial y rendimiento en la tarea. Sin embargo, nosotros creemos que, en contextos novedosos, en los que el individuo inicialmente apenas logra una baja tasa de refuerzo y en los que por definición se puede medir la persistencia (cuando los intentos de solución son fallidos), sólo se debería encontrar relación entre motivación y persistencia exploración.

\section{Método}

\section{Participantes}

La muestra analizada la conformaron 870 participantes, 274 mujeres (31.5\%) y 596 varones $(68.5 \%)$. El rango de edad de las mujeres estuvo comprendido entre los 21 y los 40 años $(M o=28 ; M d n=28)$ y el de los varones entre los 19 y los 47 $(M o=29 ; M d n=29)$. Los participantes, todos ellos licenciados universitarios, procedían de diferentes titulaciones y aspiraban a un curso de formación para en su caso acceder a un puesto de alta cualificación técnica.

\section{Materiales}

Test de banderas para la evaluación del aprendizaje discriminativo y la persistencia V2.0 (Santacreu, 2004): La prueba presenta en pantalla 10 botones organizados en forma de círculo, cada uno de los cuales muestra el dibujo de una bandera en su interior (véase Figura 1).

Figura 1. Entorno de la tarea correspondiente al Test de banderas.

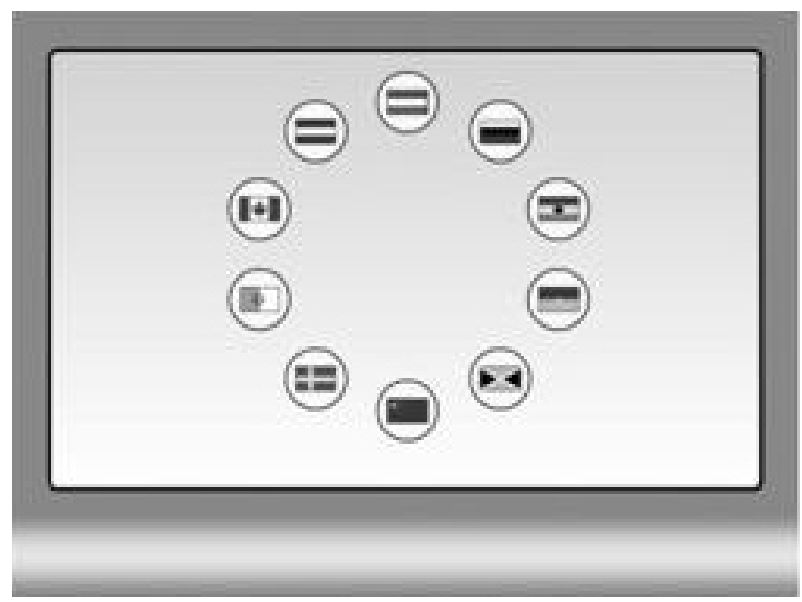

Las pantallas de instrucciones incluyen los siguientes mensajes:

Pantalla 1: "Ésta es una tarea que trata de medir la capacidad de aprendizaje en una tarea manipulativa. En las pantallas aparecerán botones que contienen símbolos (letras, banderas, números ...). Su tarea consiste en apretar SÓLO los botones necesarios para conseguir ACIERTO. Tiene que conseguir el mayor número de aciertos posibles".

Pantalla 2: Se indica el lugar en donde aparecen los mensajes de acierto y en donde debe de pulsar.

Pantalla 3: "Fíjese bien con qué botones ha conseguido el acierto. Su objetivo es conseguir acierto PERO pulsando el menor número de botones. Asegúrese que obtiene el acierto pulsando el menor número de botones".

La tarea de aprendizaje consta de dos fases, una fase de adquisición y otra de extinción.

La fase de adquisición consiste en una tarea de operante libre, en la cual se ha de pulsar sobre los 10 botones hasta obtener un reforzador, consistente en la aparición en la pantalla de un mensaje de "acierto". El reforzador se obtiene al pulsar tres veces sobre una bandera determinada (bandera clave). Al pulsar por tercera vez sobre la bandera clave aparece en pantalla el mensaje informando de que se ha conseguido un "acierto", con independencia de que en el mismo ensayo se hayan pulsado otros botones. Tras la aparición del mensaje de "acierto" daría comienzo el siguiente ensayo.

La prueba está diseñada para que en cada aplicación y para cada individuo que ejecuta la tarea la bandera clave sea distinta. Así, al inicio de la ejecución, la prueba asigna de forma aleato- 
ria la condición de "clave" a una de las banderas, la cual conservará dicha condición durante el resto de los ensayos (salvo en la fase de extinción). No obstante, en cada nuevo ensayo cambia el color de fondo de la pantalla y las banderas cambian aleatoriamente su posición.

La fase de adquisición presenta un mínimo de 15 ensayos y un máximo de 60. Esta fase termina cuando el individuo ha alcanzado un criterio de aprendizaje establecido, consistente, en este estudio, en presentar una proporción de pulsaciones sobre la bandera clave igual o superior a .50 durante, al menos, cinco ensayos.

La fase de extinción consiste en un único ensayo de un minuto de duración, durante el cual no se establece ninguna relación de contingencia entre pulsar sobre los botones y el mensaje de "acierto". Para la recogida de datos, el ensayo se ha dividido en 10 períodos de 6 segundos cada uno. Transcurrido el tiempo, e independientemente de la conducta del sujeto, se presenta en pantalla el mensaje de "acierto", dándose por concluida la tarea. Este hecho no puede afectar al comportamiento del individuo, dado que no hay más ensayos, pero permite terminar la ejecución reforzando la sesión de extinción.

\section{Indicadores}

Número de ensayos realizados durante la fase de adquisición (ENS). Constituye una medida del número de ensayos requeridos por cada sujeto para alcanzar el criterio de aprendizaje en la fase de adquisición. Un valor igual a 60 indica que el sujeto no ha cumplido dicho criterio al llegar al último ensayo de la primera fase.

Proporción de respuestas correctas durante la fase de adquisición (PRCF1). Promedio del cociente entre el número de pulsaciones realizadas sobre la bandera clave y el número total de pulsaciones realizadas correspondiente a cada uno de los ensayos de la fase de adquisición. Este indicador representa una medida del aprendizaje producido a lo largo de la fase de adquisición.

Proporción de respuestas correctas durante la fase de extinción (PRCF2). Promedio del cociente entre el número de pulsaciones realizadas sobre la bandera clave y el número total de pulsaciones realizadas en cada intervalo de la fase de extinción. Este indicador representa una medida del aprendizaje, en tanto que resistencia a la extinción, así como una medida conjunta de la persistencia obcecación-exploración. Cuando el valor tiende a 1 el individuo se muestra obcecado y cuando tiende a 0 explorador de nuevas soluciones.

Tasa de respuesta en el primer ensayo de la fase de adquisición $\left(T R_{l}\right)$. Cociente entre el número de pulsaciones realizadas durante el primer ensayo y la duración del mismo. Este indicador constituye una medida de motivación intrínseca, en tanto que su medición tiene lugar previamente a la ocurrencia de episodios de reforzamiento.
Tasa de respuesta durante la fase de adquisición (TRF1). Promedio del cociente entre el número de pulsaciones realizadas durante cada ensayo de la fase de adquisición y la duración del mismo. Este indicador constituye una medida de motivación inicial.

Tasa de respuesta durante la fase de extinción (TRF2). Promedio del cociente entre el número de pulsaciones realizadas durante cada intervalo de la fase de extinción y la duración del mismo. Este indicador constituye una medida de persistencia motivacional.

Tasa de respuestas correctas durante la fase de extinción (TRCF2). Promedio del cociente entre el número de pulsaciones realizadas sobre la bandera clave durante cada intervalo de la fase de extinción y la duración del mismo. Este indicador constituye una medida de persistencia obcecación.

Tasa de respuestas diferentes durante la fase de extinción (TRDF2). Promedio del cociente entre el número de pulsaciones realizadas sobre banderas distintas a la bandera clave durante cada intervalo de la fase de extinción y la duración del mismo. Este indicador constituye una medida de persistencia exploración.

\section{Procedimiento, registro y análisis de datos}

Los participantes completaron una batería de tests informatizados, entre los cuales se encontraban los instrumentos requeridos para el presente estudio. Durante la aplicación los participantes ejecutaron las tareas en terminales informáticos individuales dotados de filtros de pantalla que impedían observar la ejecución al resto de participantes.

El programa informático que soporta la prueba registra cada una de las acciones del participante en el proceso de evaluación. De estos datos se han extraído las frecuencias de respuesta sobre cada uno de los 10 estímulos (banderas) diferenciando si pertenecían o no a la clave configurada en cada caso y si correspondían a la fase de adquisición o de extinción. Puesto que la fase de extinción es exactamente igual en sus parámetros para todos los participantes, los índices de persistencia expresados como tasas constituyen transformaciones lineales de las variables expresadas en frecuencias, dado que la duración del ensayo de extinción es idéntica en todos los casos. En el caso de la proporción de respuestas sobre los estímulos clave y la proporción de respuestas sobre estímulos no clave, dichos índices de persistencia resultan complementarios para cada individuo.

\section{Resultados}

\section{Evaluación del aprendizaje}

En primer lugar, se ha de señalar que los cambios en la configuración de la tarea de aprendizaje adquisición-extinción, se ajustan a las pretensiones de construir un test para evaluar objetivamente la persistencia. De la muestra total $(n=870), 698$ 
individuos $(80.20 \%)$ cumplen el criterio de aprendizaje prescrito a la finalización del ensayo 60 y $366(42.10 \%)$ lo hacen durante los 15 primeros ensayos. El número medio de ensayos realizados presenta un valor de $29.695(D T=18.112)$.

Las Figuras 2 y 3 muestran la evolución del aprendizaje (tasa de respuesta -TRF1-y proporción de respuestas correctas -PRCF1- a lo largo de los 15 primeros ensayos de la fase de adquisición.

Figura 2. Evolución por ensayos de la tasa de respuesta promedio (TRF1) durante los 15 primeros ensayos de la fase de adquisición (n $=870$ ).

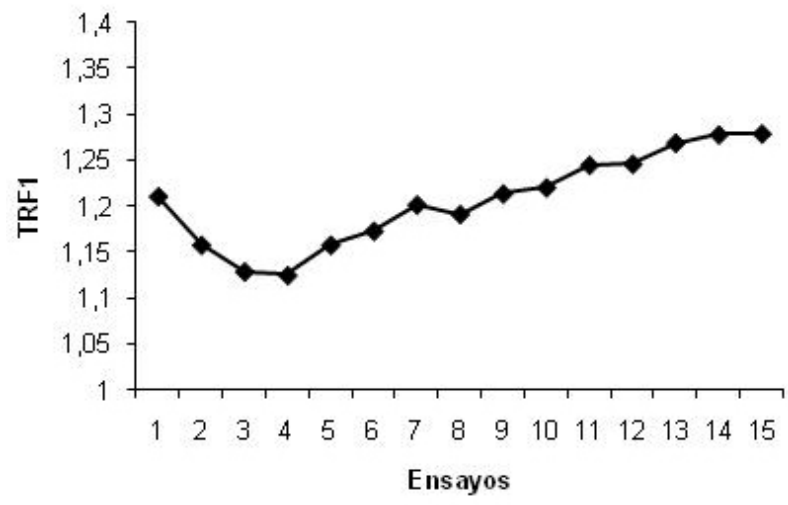

Figura 3. Evolución por ensayos de la proporción de respuestas correctas promedio (PRCF1) durante los 15 primeros ensayos de la fase de adquisición $(\mathrm{n}=870)$

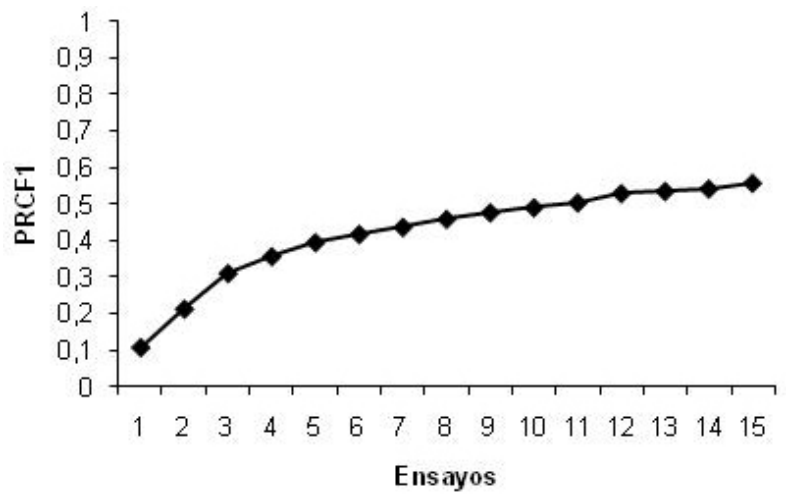

El análisis de varianza de un factor de medidas repetidas indica que la progresión observada es estadísticamente significativa, tanto en el caso de la tasa de respuesta (TRF1) $[\lambda=.867$; $\left.F(14,856)=9.415 ; p<.001 ; \eta^{2}=.133\right]$ como en el de la proporción de respuestas correctas $(P R C F 1)[\lambda=.370 ; F(14,856)$ $\left.=104.220 ; p<.001 ; \eta^{2}=.630\right]$. La tasa de respuesta correspondiente al último ensayo de la fase de adquisición $\left(T R_{15}\right)$ presenta un valor promedio de $1.279(D T=.494)$, mientras la proporción de pulsaciones correctas $\left(P R C_{15}\right)$ presenta un valor promedio de $.559(D T=.352)$.

Del mismo modo, la Figura 4 muestra la evolución del proceso de estallido y posterior extinción de la respuesta. El ANOVA de un factor de medidas repetidas arroja diferencias significativas a lo largo de la fase 2 tanto en el caso de la tasa de respuesta $($ TRF2) $[\lambda=.603 ; F(9,689)=50.330 ; p<.001$; $\left.\eta^{2}=.397\right]$, como en la tasa de respuestas correctas (TRCF2) $[\lambda$ $\left.=.383 ; F(9,689)=123.505 ; p<.001 ; \eta^{2}=.617\right]$. En el caso de la tasa de respuesta (TRF2), las comparaciones por pares (ajuste de Bonferroni) revelan un incremento significativo en el segundo intervalo con respecto al primero $(p<.001)$, así como una reducción significativa en el décimo intervalo con respecto a los intervalos $3,4,5,6$ y $8(p<.05)$. En el caso de la tasa de respuestas correctas (TRCF2), se observa una reducción progresiva a partir del segundo intervalo $(p<.001)$, la cual se estabiliza en el cuarto intervalo.

Figura 4. Evolución de la tasa de respuesta promedio (TRF2) y tasa de respuestas correctas promedio (TRCF2) por intervalos temporales (10 intervalos de 6 seg.) durante la fase de extinción $(n=698)$.

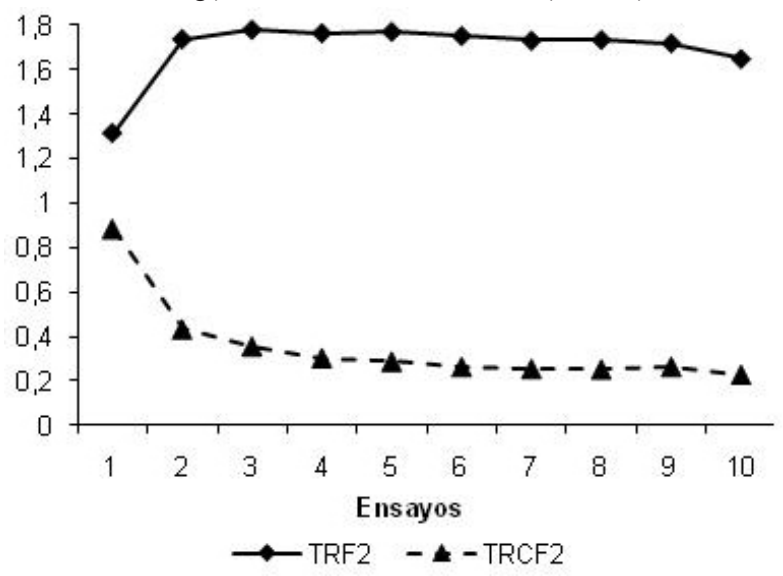

La Figura 5 muestra la evolución de la proporción de repuestas correctas durante la fase 2 (PRCF2), poniendo también de manifiesto el proceso de extinción de la respuesta. El ANOVA de un factor de medidas repetidas arroja de nuevo diferencias significativas $[\lambda=.183 ; F(9,639)=317.404 ; p<$ $\left..001 ; \eta^{2}=.817\right]$. Las comparaciones por pares ponen de relieve una reducción significativa a partir del segundo intervalo $(p<$ .001) que se estabiliza a partir del cuarto.

Figura 5. Evolución de la proporción de respuestas correctas promedio (PRCF2) por intervalos temporales (10 intervalos de 6 seg.) durante la fase de extinción $(\mathrm{n}=698)$.

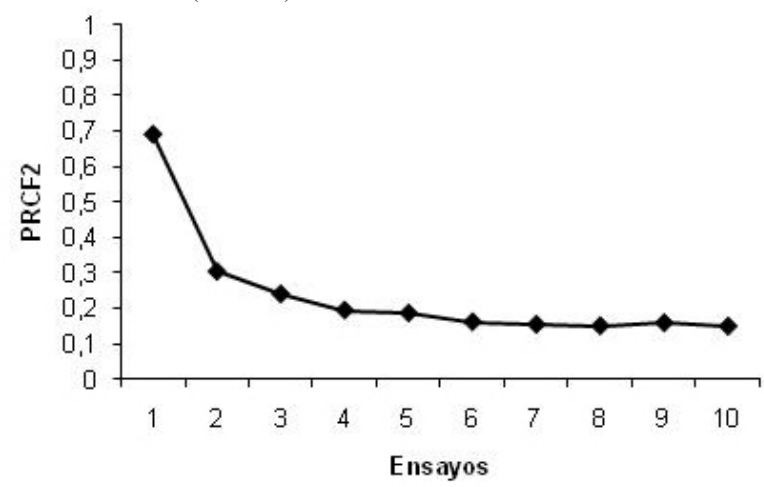


Se observa, además, durante la fase de extinción, un incremento significativo en la tasa de respuesta promedio con respecto a la fase de adquisición, tal y como pone de manifiesto el contraste sobre medias $[t(697)=-31.362 ; p<.001$; Cohen's $d=1.384]\left(M d_{1}=1.233, D T=.376 ; M d_{2}=1.696, D T=.588\right)$. Como era de esperar, durante la fase de extinción se observan también diferencias significativas entre la tasa de respuesta (TRF2) y la tasa de respuestas correctas promedio (TRCF2) $[t$ $(697)=65.148 ; p<.001$; Cohen's $d=.3 .289]\left(M d_{1}=1.696, D T\right.$ $\left.=.588 ; M d_{2}=.352, D T=.158\right)$.

\section{Evaluación de la persistencia}

El estudio del comportamiento durante la fase de extinción de la tarea permite valorar el funcionamiento de la prueba como test objetivo de la persistencia. La Tabla 1 muestra los estadísticos descriptivos correspondientes a cada uno de los indicadores de persistencia durante el período de test (extinción). El resultado del estadístico Kolmogorov-Smirnov sugiere rechazar la hipótesis de normalidad en todos los casos [TRF2: K-S (698) $=.062, p<.001 ;$ PRCF $2: K-S(698)=.118, p<.001 ;$ TRCF 2 : $K-S(698)=.112, p<.001 ; T R D F 2: K-S(698)=.049, p<.001]$.

Tabla 1. Estadísticos descriptivos correspondientes a los indicadores de persistencia motivacional, persistencia obcecación y persistencia exploración $(\mathrm{N}=698)$.

\begin{tabular}{lcccccccc}
\hline & Md & $D T$ & Min & Max & Asimetria & ET & Curtosis & ET \\
\hline TRF2 & 1.696 & .588 & .230 & 3.800 & .655 & .093 & .619 & .185 \\
PRCF2 & .239 & .102 & .06 & .83 & 1.387 & .093 & 2.580 & .185 \\
TRCF2 & .352 & .158 & .100 & 1.170 & 1.387 & .093 & 2.956 & .185 \\
TRDF2 & 1.343 & .545 & .130 & 3.270 & .560 & .093 & .355 & .185 \\
\hline
\end{tabular}

Nota : TRF2 = Tasa de respuesta durante la fase de extinción; PRCF2 = Proporción de respuestas correctas durante la fase de extinción; TRCF2 = Tasa de respuestas correctas durante la fase de extinción; TRDF2 = Tasa de respuestas diferentes durante la fase de extinción.

Finalmente, se calculó el valor del estadístico alfa de Cronbach correspondiente a los índices de persistencia a partir de las medidas registradas en cada uno de los 10 intervalos que conforman la fase de extinción. Los resultados obtenidos presentan valores de .928 , en el caso de TRF2, .683 en el de PRCF2, .580 en el de TRCF2, y .905 en el de TRDF2.

\section{Relación entre medidas de persistencia}

En la Tabla 2 se muestran los valores del coeficiente de correlación de Pearson correspondientes a los distintos indicadores de persistencia y motivación.

Como puede apreciarse, la relación entre la persistencia obcecación $(T R C F 2)$ y persistencia exploración $(T R D F 2)$ es relativamente baja, aunque significativa $(r=.131, p<.001)$. Sin embargo, la relación de cada una de ellas con la persistencia motivacional (TRF2) presenta valores de $.964(p<.001)$, en el caso de la persistencia exploratoria, y $.400(p<.001)$, en el caso de la persistencia obcecación. Como era de esperar, la pro- porción de pulsaciones correctas en fase de extinción (PRCF2) presenta valores de correlación similares con los índices mencionados, aunque de signo positivo con TRCF2 $(r=.574, p$ $<.001)$, y signo negativo con TRF2 $(r=-.403, p<.001)$ y TRDF2 $(r=-.602, p<.001)$. Por otra parte, tal y como se había hipotetizado, el indicador de persistencia motivacional (TRF2) presenta valores de correlación elevados con los indicadores de motivación inicial $(T R F 1)(r=.759 ; p<.001)$ y motivación intrínseca $\left(T R_{1}\right)(r=.605 ; p<.001)$. Igualmente acorde con lo esperado, el indicador de motivación inicial (TRF1) presenta un valor de correlación mayor con el indicador de persistencia exploración $(T R D F 2)(r=.751, p<.001)$ que con el indicador de persistencia obcecación $(T R C F 2)(r=.238, p<.001)$, siendo estas diferencias estadísticamente significativas $[t$ (695) $=15.966 ; z=13.636 ; p<.001)$. Del mismo modo, el indicador de motivación intrínseca $\left(T R_{l}\right)$ presenta un valor de correlación mayor con el indicador de persistencia exploración (TRDF2) $(r=.615, p<.001)$ que con el indicador de persistencia obcecación (TRCF2) $(r=.131, p<.001)$, siendo de nuevo las diferencias estadísticamente significativas $[t(695)=12.379 ; z=$ $11.160 ; p<.001)$.

Tabla 2. Matriz de correlaciones de Pearson entre los indicadores promedio de persistencia TRF2, PRCF2, TRCF2 y TRDF2 y motivación TRF1, $\mathrm{TR}_{1}(\mathrm{n}=698)$.

\begin{tabular}{lcccccc}
\hline & 1 & 2 & 3 & 4 & 5 & 6 \\
\hline 1. $T R F 2$ & 1 & $-.403^{* *}$ & $.400^{* *}$ & $.964^{* *}$ & $.759^{* *}$ & $.605^{* *}$ \\
2. $P R C F 2$ & & 1 & $.574^{* *}$ & $-.602^{* *}$ & $-.420^{* *}$ & $-.339^{* *}$ \\
3. $T R C F 2$ & & & 1 & $.143^{* *}$ & $.238^{* *}$ & $.131^{* *}$ \\
4. $T R D F 2$ & & & & 1 & $.751^{* *}$ & $.615^{* *}$ \\
5. $T R F 1$ & & & & & 1 & $.611^{* *}$ \\
6. $T R_{I}$ & & & & & & 1 \\
\hline
\end{tabular}

Nota: TRF2 = Tasa de respuesta durante la fase de extinción; PRCF2 = Proporción de respuestas correctas durante la fase de extinción; TRCF2 $=$ Tasa de respuestas correctas durante la fase de extinción; TRDF2 = Tasa de respuestas diferentes durante la fase de extinción; TRF1 = Tasa de respuesta durante la fase de adquisición; $\mathrm{TR}_{1}=$ Tasa de respuesta en el primer ensayo de la fase de adquisición.

$* * p<.001$

\section{Discusión}

Los resultados obtenidos en el presente trabajo permiten concluir que un elevado porcentaje de participantes aprende la relación de contingencia establecida por la prueba. En concreto, un $80.20 \%$ de la muestra alcanza el criterio de aprendizaje en los 60 ensayos y, por tanto, en estos casos, se podría evaluar diferencialmente la persistencia obcecación versus exploración. De acuerdo con lo esperado, se observa un incremento progresivo a lo largo de los 15 primeros ensayos en lo relativo a la proporción de respuestas correctas y la tasa de respuesta.

Del mismo modo, durante la fase de test, se observa un incremento inicial en la tasa de respuesta, fruto del estallido de respuesta característico del inicio del período de extinción, así como una reducción progresiva en la proporción de respuestas correctas como resultado del cese del reforzamiento. 
Cumplidas las condiciones previas necesarias para la evaluación de los tres componentes de la persistencia, el análisis descriptivo e histogramas correspondientes a los distintos indicadores calculados revelan suficiente variabilidad en la distribución de frecuencias. Cabe considerar este resultado síntoma de que la prueba de evaluación diseñada, a diferencia de versiones anteriores, permite manifestar la variabilidad interindividual en el comportamiento persistente (Santacreu et al., 2002). Los índices de consistencia interna correspondientes a los indicadores expresados en tasas presentan valores entre moderados y altos: .928 en el caso de persistencia motivacional, .580 en el caso de persistencia obcecación, .905 en el de persistencia exploración y un valor de .683 en el caso de la proporción de respuestas correctas en fase de extinción. Dichos valores pueden ser considerados indicativos de una elevada precisión en la medición cuando se analiza, como en este caso, el primer minuto de extinción de la respuesta

De acuerdo con el análisis de correlaciones, el componente general de persistencia motivacional presenta una relación positiva y significativa con los componentes de obcecación (.400) y exploración (.964), expresados en tasas, y una correlación de -.403 con el índice expresado en proporción. En cambio, la persistencia exploración se muestra relativamente independiente de la persistencia obcecación (.143), dato que permite sostener que obcecación y exploración suponen aspectos distintos de la persistencia de los individuos.

Conforme a lo hipotetizado, la medida de persistencia motivacional presenta una correlación elevada, positiva y significativa con el indicador de motivación inicial $(r=759, p<$ $.001)$, así como con el indicador de motivación intrínseca $(r$ $=.605, p<.001)$. Estos resultados vienen a respaldar los ya observados en estudios anteriores, según los cuales la motivación inicial se muestra predictora de la persistencia del comportamiento (Allen, 1999; Cardoza, 1991; Deci, Koestner y Ryan, 1999; Matsumoto y Obana, 2001; Pelletier, Fortier, Vallerand y Briere, 2001; Ramage, 1990; Vallerand y Bissonnette, 1992). No obstante, se ha de añadir que, de acuerdo con los planteamientos expuestos en la introducción, la relación de la motivación con la persistencia exploración es mayor que con persistencia obcecación. En este sentido, cabe concluir que en contextos inciertos, en los cuales el individuo desconoce la relación de contingencia exacta que determina la obtención de reforzadores, la motivación inicial únicamente se relaciona con la persistencia en el comportamiento exploratorio.

A pesar de que el momento en que tiene lugar el aprendizaje tiene una baja influencia sobre las medidas de persistencia, tal y como se deriva de la observación de las correlaciones y el tamaño del efecto, cabe plantear como futuro objetivo de investigación reducir aún más dicha influencia. Para ello, se propone simplificar la relación de contingencia que determina el reforzamiento a un programa $R F-2$, siendo de esperar una reducción de la variabilidad en el número de ensayos requeridos para alcanzar el criterio de rendimiento. Cabe esperar, de este modo, que una mayor homogeneidad en la duración del período de aprendizaje derive en un mayor control sobre su influencia en el comportamiento durante la fase de extinción.

A modo de conclusión podemos afirmar que la medición de la persistencia se ha mostrado plenamente viable mediante la utilización de una tarea de operante libre con fase de adquisición-extinción. La prueba diseñada en esta ocasión ha mostrado un mejor cumplimiento de los requerimientos necesarios para la evaluación de la persistencia que las pruebas empleadas en estudios anteriores (Hernández, García-Leal, Rubio y Santacreu, 2004; Santacreu y García-Leal, 2000). Además, se ha registrado una nueva medida de persistencia que permite poner de manifiesto la independencia entre dos aspectos diferentes de la misma, los componentes de obcecación y exploración, resultado que permite apoyar su consideración como estilos interactivos independientes o al menos como extremos de una dimensión. Se abre aquí un camino de cara a la obtención de medidas de validez de constructo y de criterio para ambos indicadores, vislumbrándose a priori, posibles implicaciones de cara a la predicción de la capacidad de adaptación de los individuos a determinados entornos que requieran de la persistencia en el comportamiento.

\section{Referencias}

1. Allen, D. (1999). Desire to finish college: An empirical link between motivation and persistence. Research in Higher Education, 40, 461-485.

2. Balleine, B. W., Garner, C., González, F. y Dickinson, A. (1995). Motivational control of heterogeneous instrumental chains. Journal of Experimental Psychology: Animal Behavior, 21, 203-217. doi:10.1037/0097-7403.21.3.203

3. Berlyne, D. I. (1960). Motivational problems raised by exploratory and epistemic behavior. En S. Koch (Ed.), Psychology: A study of a science (Vol. 5) (pp. 284-364). McGraw-Hill. doi:10.1037/10040-006

4. Campbell, J. P. y Pritchard, D. (1983). Motivation theory in industrial and organizational psychology. En M. D. Dunnette (Ed.), Handbook of industrial and organizational psychology (pp. 63-130). Chicago: Rand McNally.

5. Cardoza, D. (1991). College attendance and persistence among Hispanic women: An examination of some contributing factors. Sex Roles, 24, 133-146. doi:10.1007/ BF00288887

6. Catania, A. C. (1992). Learning. Elglewood Cliffs, NJ: Prentice Hall.

7. Cattell, R. B. (1979). Personality and learning theory. New York: Springer Pub. Co.

8. Dam, G. y Körding, K. (2009). Exploration and exploitation during sequential search. Cognitive Science, 33, 530541. doi:10.1111/j.1551-6709.2009.01021.x

9. Daw, N., Niv, Y. y Dayan, P. (2005). Uncertainty-based competition between prefrontal and dorsolateral striatal 
systems for behavioral control. Nature Neuroscience, 8, 1704-1711. doi:10.1038/nn1560

10. Deci, E. L., Koestner, R. y Ryan, R. M. (1999). A metaanalytic review of experiments examining the effects of extrinsic rewards on intrinsic motivation. Psychological Bulletin, 125, 627-668. doi:10.1037/0033-2909.125.6.627

11. Feather, N. T. (1962). The study of persistence. Psychological Bulletin, 59, 94-115. doi:10.1037/h0042645

12. Hernández, J. M., García-Leal, O., Rubio, V. y Santacreu, J. (2004). La persistencia en el estudio conductual de la personalidad. Psicothema, 16, 39-44.

13. Honig, W. H. y Staddon, J. E. R. (1997). Handbook of operant behavior. New Jersey: Prentice Hall.

14. Hutt, C. (1970). Specific and diverse exploration. En H. W. Reese y L. P. Lipsilt (Eds.), Advances in child development and behavior (Vol. 5) (pp. 119-180). New York: Academic Press.

15. Jenkins, W. O. y Stanley, J. C. (1950). Partial reinforcement: A review and critique. Psychological Bulletin, 47, 193-234. doi:10.1037/h0060772

16. Joyce, J. y Chase, P.(1990). Effects of response variability on the sensitivity of rule-governed behavior. Journal of the Experimental Analysis of Behavior, 54, 251-262. doi:10.1901/jeab.1990.54-251

17. Kaelbling, L. P., Littman, M. L. y Moore, A. W. (1996). Reinforcement learning: A survey. Journal of Artificial Research, 4, 237-287.

18. Katila, R. y Ahuja, G. (2002). Something old, something new: A longitudinal study of search behavior and new product introduction. Academy of Management Journal, 45, 1183-1194. doi: $10.2307 / 3069433$

19. Lewis, D. J. (1960). Partial reinforcement: A selective review of the literature since 1950. Psychological Bulletin, 57, 1-28. doi:10.1037/h0040963

20. Luciano, M. C. (1993). La conducta verbal a la luz de las recientes investigaciones. Su papel sobre otras conductas verbales y no verbales. Psicothema, 5, 351-374.

21. March, J. G. (1991). Exploration and exploitation in organizacional learning. Organization Science, 2, 71-87. doi:10.1287/orsc.2.1.71

22. Martínez, H. y Tamayo, R. (2005). Interactions of contingencies, instructional accuracy, and instructional history in conditional discrimination. The Psychological Record, 55, 633-646.
23. Matsumoto, M. y Obana, Y. (2001). Motivational factors and persistence in learning Japanese as a foreign language. New Zealand Journal of Asian Studies, 3, 59-86.

24. McAdams, D. P. y Pals, J. L. (2007). The role of theory in personality research. En R. Robins, C. Fraley y R. Krueger (Eds.), Handbook of research methods in personality psychology (pp. 3-20). New York: Guilford Press.

25. Moreno, R. y Hunziker, M. H. (2008). Behavioral variability: a unified notion and some criteria for experimental analysis. Revista Mexicana de Análisis de la Conducta, 34, 135-145.

26. Neuringer, A. J. (2002) Operant variability: Evidence, functions, and theory Psychonomic Bulletin \& Review, 9, 672-705. doi:10.3758/BF03196324

27. Pelletier, L. G., Fortier, M. S., Vallerand, R. J. y Briere, N. M. (2001). Associations amog perceived autonomy support, forms of self-regulation, and persistence: A prospective study. Motivation and Emotion, 25, 279-306. doi:10.1023/A:1014805132406

28. Ramage, K. (1990). Motivational factors and persistence in foreign language study. Language Learning, 40, 189-219. doi:10.1111/j.1467-1770.1990.tb01333.x

29. Rubio, V. y Santacreu, J. (2004). TRAS-I: Test adaptativo informatizado para la evaluación del razonamiento secuencial y la inducción. Madrid: TEA.

30. Santacreu, J. y García-Leal, O. (2000). La utilización de test comportamentales informatizados en el estudio de la personalidad: La evaluación de la persistencia. Psicothema, 12, 93-98.

31. Santacreu, J., Hernández, J. M., Adarraga, P. y Márquez, M. O. (2002). La personalidad en el marco de una teoría del comportamiento humano. Madrid: Ediciones Pirámide.

32. Sutton, R. S. y Barto, A. G. (1998). Reinforcement learning. Cambridge, MA: MIT Press.

33. Vollmeyer, R. y Rheinberg, F. (2000). Does motivation affect performance via persistence? Learning and Instruction, 10, 293-309. doi:10.1016/S0959-4752(99)00031-6

Fecha de recepción: 9 de marzo de 2011 Fecha de aceptación: 12 de abril de 2011 